\title{
Healthcare management information system usage: the moderating effect of psychological health status
}

\author{
$\left[\right.$ Shi-Qi Xiao ${ }^{1}$, Shih-Jung Hsiao ${ }^{2}$, Cheng-Peng Kuo ${ }^{3}$, Chun-Yu Tien ${ }^{4}$, I-Chiu Chang ${ }^{5}$ ]
}

\begin{abstract}
In this study, the moderating effect of psychological health status on the usage intention of Health Management Information Systems (HMIS) by nurses was observed. The effectiveness of HMIS depends on the willingness of people to use these systems. Nurses play a key role in healthcare, and their psychological health status may influence their usage intention of HMIS. Empirical data to assess the hypotheses in this study were obtained using a survey method. Data from a sample of nurses in Northeast China and Taiwan were collected in 2013 by mail using research instruments based on our research framework. The sample size was $\mathbf{1 , 5 6 3}$ nurses and the valid response rate was $\mathbf{5 5 . 8 \%}$. The results showed that nurses' psychological health status was a significant moderator in determining nurses' usage intention of HMIS. Nurses with positive psychological health were seen to be willing to use HMIS due to the perceived usefulness of HMIS. Nurses with negative psychological health were not seen to be willing to use HMIS due to some perceived difficulties in the ease of use of HMIS. Nurses' perception of the ease of use of HMIS should be strengthened, as this will help to improve the effectiveness of their work. The nursing staff shortage has led to workassociated psychological stress; however, the use of HMIS may help to protect and maintain the psychological health of nurses. These results suggest that protecting nurses' psychological health status is important, since it can reduce resistance from nurses when a new system is implemented and therefore enhance the efficiency of nurses.
\end{abstract}

Keywords-Nurses, Psychological health status, Health Management Information Systems (HMIS), Usage intention, Technology acceptance model (TAM)

\section{Shi-Qi Xiao}

College: Shengjing Hospital of China Medical University

Country: China

Shih-Jung Hsiao

College: National Chung Cheng University

Country: Taiwan

Cheng-Peng Kuo

College: National Chung Cheng University

Country: Taiwan

Chun-Yu Tien

College: National Chung Cheng University Country: Taiwan

I-Chiu Chang

College: National Chung Cheng University

Country: Taiwan 